\title{
Performance analysis of polymer optical fibre based Fabry-Perot sensor formed by two uniform Bragg gratings
}

\author{
A. Pospori*, D. J. Webb \\ Aston Institute of Photonic Technologies, Aston University, Aston Triangle, Birmingham, B7 4ET, \\ United Kingdom \\ *a.posporis@aston.ac.uk; orcid.org/0000-0003-4866-1361
}

\begin{abstract}
The stress sensitivity of polymer optical fibre (POF) based Fabry-Perot sensors formed by two uniform Bragg gratings with finite dimensions is investigated. POF has received high interest in recent years due to its different material properties compared to its silica counterpart. Biocompatibility, a higher failure strain and the highly elastic nature of POF are some of the main advantages. The much lower Young's modulus of polymer materials compared to silica offers enhanced stress sensitivity to POF based sensors which renders them great candidates for acoustic wave receivers and any kind of force detection. The main drawback in POF technology is perhaps the high fibre loss. In a lossless fibre the sensitivity of an interferometer is proportional to its cavity length. However, the presence of the attenuation along the optical path can significantly reduce the finesse of the Fabry-Perot interferometer and it can negatively affect its sensitivity at some point. The reflectivity of the two gratings used to form the interferometer can be also reduced as the fibre loss increases. In this work, a numerical model is developed to study the performance of POF based Fabry-Perot sensors formed by two uniform Bragg gratings with finite dimensions. Various optical and physical properties are considered such as grating physical length, grating effective length which indicates the point where the light is effectively reflected, refractive index modulation of the grating, cavity length of the interferometer, attenuation and operating wavelength. Using this model, we are able to identify the regimes in which the PMMA based sensor offer enhanced stress sensitivity compared to silica based one.
\end{abstract}

Keywords: POF, polymer fibre, Fabry-Perot, sensors, stress sensitivity, Bragg gratings, modelling

\section{INTRODUCTION}

The different material properties of polymer optical fibre (POF) compared to silica optical fibre (SOF) is the reason for the high attention received from the research community ${ }^{1}$. Polymers have much lower Young's modulus ${ }^{2,3}$ and higher failure strain ${ }^{4}$. Therefore, POF based sensors can offer enhanced $\operatorname{strain}^{5}$, stress ${ }^{6}$ and pressure ${ }^{7}$ sensitivity compared with the SOF based sensors and this attribute renders them great candidates for acoustic wave receivers ${ }^{8}$ and any kind of force detection. They are biocompatible ${ }^{9}$ and some materials such as PMMA can absorb water ${ }^{10,11}$ which leads to applications in humidity monitoring. In addition, polymers' larger thermo-optic coefficients ${ }^{12-14}$ offer enhanced sensitivity in temperature monitoring applications ${ }^{15,16}$.

One of the main drawbacks in POF technology is the high fibre loss. In the absence of attenuation, the stress sensitivity of a Fabry-Perot interferometric sensor is proportional to its cavity length. However, if attenuation is present along the optical path, the finesse of a Fabry-Perot interferometer can be significantly reduced as the cavity length increases. Therefore, the fibre attenuation factor should be taken into account when POF based Fabry-Perot sensors are constructed and the optimum cavity length for maximum sensitivity should be evaluated. Constructing cavity lengths beyond that critical point will not only create a larger sensor but it will also diminish the sensor's performance.

In this paper, a numerical model has been developed with MATLAB tools in order to study the performance of POF based Fabry-Perot sensors formed by two uniform fibre Bragg gratings (FBGs) with finite dimensions. FBGs act as mirrors and can form an interferometric cavity. In this model, various optical and physical properties are considered such as operating wavelength, fibre mechanical and optical properties, physical lengths and refractive index modulation of the FBGs, cavity length of the interferometer and attenuation in the cavity optical path. The attenuation factor is also added in the coupled-mode equations used to describe the grating characteristics. In this study, we analyse the stress sensitivity of Fabry-Perot sensors as the cavity length increases. Using this model, we are able to find the regimes where POF based sensors can offer enhanced stress sensitivity compared to their silica counterparts.

Micro-Structured and Specialty Optical Fibres IV, edited by Kyriacos Kalli,

Alexis Mendez, Proc. of SPIE Vol. 9886, 98861F · (c) 2016 SPIE

CCC code: $0277-786 X / 16 / \$ 18 \cdot$ doi: $10.1117 / 12.2227481$

Proc. of SPIE Vol. $988698861 \mathrm{~F}-1$ 


\section{ANALYSIS AND MODELLING}

\subsection{Reflection and transmission coefficients of Bragg grating}

Bragg gratings act as mirrors and can be used to form a Fabry-Perot cavity. Their reflection and transmission coefficients will contribute to the Fabry-Perot's finesse. The analytic solution of the propagating waves in FBG is described using the coupled-wave theory ${ }^{17}, 18$. In our case where we have a considerably high fibre loss, the attenuation factor is included in the equations for more accurate results. Therefore, the reference wave $R$ and the signal wave $S$ along the grating position $z$ become

$$
\begin{gathered}
\frac{d R}{d z}=-j \kappa * S e^{j \psi z}-\frac{a}{2} R, \\
\frac{d S}{d z}=j \kappa R e^{-j \psi z}+\frac{a}{2} S,
\end{gathered}
$$

where $\alpha$ is the attenuation, $\psi$ is the off-Bragg vector and $\kappa$ is the coupling coefficient between the waves which given by

$$
\kappa=\frac{\Delta n \pi}{\lambda_{B}}
$$

where $\lambda_{B}$ is the Bragg wavelength and $\Delta n$ is the refractive index modulation used to form the Bragg grating periodic structure. In our case, we will deal with uniform gratings where $\lambda_{B}$ and $\Delta n$ are constant. The off-Bragg condition $\psi$ can be expressed as

$$
\psi=4 n \pi\left(\frac{1}{\lambda}-\frac{1}{\lambda_{B}}\right),
$$

where $\lambda$ is the free space wavelength and $n$ is the effective refractive index of the fibre. In order to numerically solve the analytic equations (1) and (2), the boundary conditions should be set. Considering a grating with a length $L_{g}$, the boundary conditions are

$$
\begin{gathered}
R(0)=1, \\
S\left(L_{g}\right)=0,
\end{gathered}
$$

where expression (5) assumes a unity input and (6) assumes that there is not a signal wave at the far end of the grating. The amplitude diffraction efficiency $\eta$ of the grating can be defined as

$$
\eta(z)=\frac{S(z)}{R(z)} .
$$

Differentiating (7) leads to

$$
\frac{d \eta}{d z}=\frac{1}{R} \frac{d S}{d z}-\frac{S}{R^{2}} \frac{d R}{d z} .
$$

Substituting equations (1) and (2) to (8) leads to a Riccati equation, which can be solved to find the diffraction efficiency $\eta$ by integrating numerically from the far end of the grating to the near end: 


$$
\frac{d \eta}{d z}=j \kappa e^{-j \psi z}+\alpha \eta+j \kappa * e^{j \psi z} \eta^{2}
$$

The power reflection coefficient of the grating equals

$$
r=\eta \eta^{*}
$$

Combining equations (1) and (2), the transmission wave of the grating can be also expressed as

$$
\frac{d R^{2}}{d z^{2}}=\left(\kappa^{2}+\frac{a^{2}}{4}\right) R,
$$

which can be solved by using the boundary conditions:

$$
\begin{gathered}
R(0)=1, \\
\left.\frac{d R}{d z}\right|_{z=L_{g}}=-\frac{a}{2} R\left(L_{g}\right) .
\end{gathered}
$$

The power transmission coefficient can be expressed as

$$
t=R R^{*}
$$

\subsection{Effective length of Bragg grating}

Another parameter to consider for accurate results is the effective length of the grating, which shows the effective penetration depth of the propagating light before being reflected back. The distance between the two gratings and their effective lengths are needed to define the actual cavity length of the interferometer (Figure 1). If the reflection coefficient of the grating is at very low levels, the effective length is half of the grating physical length ${ }^{19}$. However, as the grating reflectivity increases, most of the light is reflected back from the first grating planes and we can translate this to a shorter effective length.

The effective length of the grating or the penetration depth of the light coincides with the group length ${ }^{19}$ and equals

$$
L_{e f f}=\frac{v_{g} \tau}{2}
$$

where $v_{g}=c / n_{g}$ is the group velocity ( $n_{g} \approx n$ is assumed) and $\tau$ is the group delay given by

$$
\tau=-\frac{\lambda^{2}}{2 \pi c} \frac{d \phi}{d \lambda},
$$

where $c$ is the speed of light and $\varphi$ is the phase of the diffraction efficiency $\eta$ which is defined in equation (9). 


\subsection{Fabry-Perot interferometer}

Two uniform Bragg gratings which act as partially reflective mirrors are used to form the Fabry-Perot etalon as depicted in Figure 1. In that cavity, a constructive interference occurs when the transmitted beams are in phase. The electric field shown in Figure 1 can be expressed as

$$
E_{1}=\sqrt{t_{1}} E_{0}+\sqrt{r_{1}} E_{4}, \quad E_{2}=E_{1} e^{j k L} e^{-\frac{\alpha}{2} L}, \quad E_{3}=\sqrt{r_{2}} E_{2}, \quad E_{4}=E_{3} e^{j k L} e^{-\frac{\alpha}{2} L}, \quad E_{t}=\sqrt{t_{2}} E_{2},
$$

where $E_{0}$ is the input electric field normal to FBG 1, $\alpha$ is the attenuation in the cavity, $r$ and $t$ are the reflection and transmission coefficients for each grating and $k$ is the wavenumber which can be expressed as

$$
k=\frac{2 \pi n}{\lambda}
$$

Combining the expressions (16), the transmitted electric field $E_{t}$ coming out of FBG 2 can be written as

$$
E_{t}=\frac{\sqrt{t_{1}} \sqrt{t_{2}} E_{0} e^{j k L} e^{-\frac{a}{2} L}}{1-\sqrt{r_{1}} \sqrt{r_{2}} e^{2 j k L} e^{-a L}}
$$

For a unity input field $\left(E_{0}=1\right)$ the transmission intensity $\left(I_{t}=E_{t} E_{t}{ }^{*}\right)$ of the Fabry-Perot interferometer equals

$$
I_{t}=\frac{t_{1} t_{2} e^{-a L}}{1-\left(2 \cos (2 k L) \sqrt{r_{1}} \sqrt{r_{2}} e^{-a L}\right)+r_{1} r_{2} e^{-2 a L}} .
$$

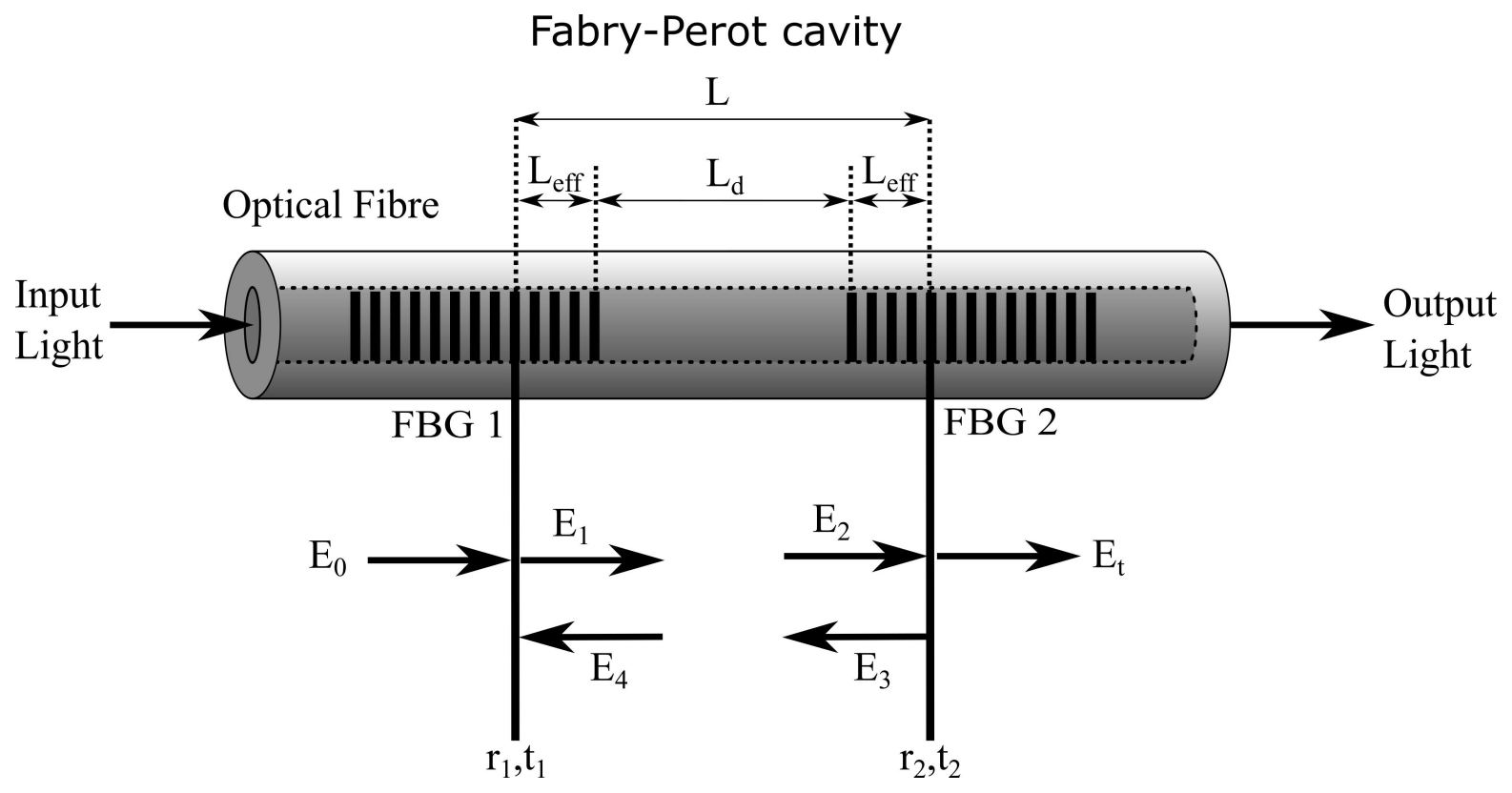

Figure 1: Fabry-Perot formed by two uniform fibre Bragg gratings 


\subsection{Modelling methodology}

First, the reflection and transmission coefficients $\left(r_{1,2}\right.$ and $\left.t_{1,2}\right)$ and the effective length $\left(\mathrm{L}_{\text {effl, }}\right)$ for each grating is calculated at the Bragg wavelength. For illustration purposes only, in our model we used two different grating lengths $L_{g}=3 \mathrm{~mm}$ and $L_{g}=2 \mathrm{~mm}$ with refractive index change $\Delta n=2 * 10^{-4}$. The total cavity length $L$ of the interferometer is defined as

$$
L=L_{\text {eff } 1}+L_{d}+L_{\text {eff } 2} \text {, }
$$

where $L_{d}$ is the distance between the two gratings (Figure 1). The transmission intensity $I_{t}$ of the Fabry-Perot expressed in equation (19) can now be numerically solved. The strain sensitivity of the Fabry-Perot sensor can be determined by differentiating its transmission intensity with respects to strain:

$$
\frac{d I_{t}}{d \varepsilon}=\frac{\partial I_{t}}{\partial n} \frac{\partial n}{\partial \varepsilon}+\frac{\partial I_{t}}{\partial L} \frac{\partial L}{\partial \varepsilon}
$$

If a homogeneous and isotropic elastic material is considered, the refractive index change due to $\operatorname{strain}^{20}$ is

$$
\frac{\partial n}{\partial \varepsilon}=\frac{n^{3}\left(\mu P_{11}+(\mu-1) P_{12}\right)}{2},
$$

where $P_{11}$ and $P_{12}$ are the Pockels coefficients and $\mu$ is the Poisson's ratio of the material. The term $\partial L / \partial \varepsilon$ in equation (21) is actually the total cavity length $L$. The remaining terms can be found by differentiating equation (19) with respect to the variables in the expression.

PMMA strain coefficients provide to their sensors approximately $14 \%$ more strain sensitivity compared to silica based sensors $^{21}$. However, the stress sensitivity of PMMA based sensors is considerably higher. The much lower Young's modulus of PMMA ${ }^{3}(2.8 \mathrm{GPa})$ compared to silica ${ }^{21}(73 \mathrm{GPa})$ provides approximately 25 times more elasticity to the fibre. The relation of strain $\varepsilon$, stress $\sigma$ and Young's modulus $E$ is:

$$
E=\frac{\sigma}{\varepsilon}
$$

Therefore the stress sensitivity of the Fabry-Perot interferometer can be defined as

$$
\frac{d I_{t}}{d \sigma}=\frac{d I_{t}}{d \varepsilon} \frac{1}{E}
$$

The typical Pockel's coefficients ${ }^{22,23}$, Poisson ratio ${ }^{21,24}$, attenuation ${ }^{25}$ and refractive indices ${ }^{26,27}$ for $\lambda=850 \mathrm{~nm}$ and $\lambda=1550 \mathrm{~nm}$ of PMMA and silica optical fibres are shown in table 1 .

Table 1: Material properties of PMMA and fused silica

\begin{tabular}{|l|c|c|}
\cline { 2 - 3 } \multicolumn{1}{c|}{} & PMMA & Fused silica \\
\hline $\mathrm{P}_{11}$ & 0.300 & 0.113 \\
\hline $\mathrm{P}_{12}$ & 0.297 & 0.252 \\
\hline$\mu$ & 0.345 & 0.170 \\
\hline$\alpha(\lambda=850 \mathrm{~nm})$ & $2000 \mathrm{~dB} / \mathrm{km}$ & $1.0 \mathrm{~dB} / \mathrm{km}$ \\
\hline$\alpha(\lambda=1550 \mathrm{~nm})$ & $100000 \mathrm{~dB} / \mathrm{km}$ & $0.2 \mathrm{~dB} / \mathrm{km}$ \\
\hline $\mathrm{n}(\lambda=850 \mathrm{~nm})$ & 1.4840 & 1.4525 \\
\hline $\mathrm{n}(\lambda=1550 \mathrm{~nm})$ & 1.4710 & 1.4440 \\
\hline
\end{tabular}




\section{RESULTS}

The reflection and transmission coefficients and the effective lengths of FBGs with grating physical lengths $L_{g}=3 \mathrm{~mm}$ in PMMA and silica optical fibres are shown in Table 2 for both $850 \mathrm{~nm}$ and $1550 \mathrm{~nm}$ wavelengths. The respective values for grating lengths $L_{g}=2 \mathrm{~mm}$ are shown in Table 3. Longer grating lengths result in higher reflection coefficients due to increased number of Bragg reflectors. When the Bragg wavelength is at $1550 \mathrm{~nm}$, where the period of the grating is larger compared to an $850 \mathrm{~nm}$ grating, there are fewer Bragg reflectors when the grating physical length is kept fixed. Fewer Bragg reflectors result in a lower grating reflection coefficient. In addition, PMMA FBGs exhibit less reflection if they are operating in the $1550 \mathrm{~nm}$ wavelength region due to the higher attenuation compared to the $850 \mathrm{~nm}$ wavelength region (Table 1).

Table 2: FBGs characteristics for $L_{g}=3 \mathrm{~mm}$

\begin{tabular}{|c|c|c|c|}
\cline { 3 - 4 } \multicolumn{2}{c|}{} & PMMA FBG & Silica FBG \\
\hline \multirow{3}{*}{$\lambda=850 \mathrm{~nm}$} & $\mathrm{r}$ & 0.9531 & 0.9537 \\
\cline { 2 - 4 } & $\mathrm{t}$ & 0.0463 & 0.0463 \\
\cline { 2 - 4 } & $\mathrm{L}_{\text {eff }}(\mathrm{mm})$ & 0.6605 & 0.6606 \\
\hline \multirow{3}{*}{$\lambda=1550 \mathrm{~nm}$} & $\mathrm{r}$ & 0.6704 & 0.7031 \\
\cline { 2 - 4 } & $\mathrm{t}$ & 0.2830 & 0.2969 \\
\cline { 2 - 4 } & $\mathrm{L}_{\text {eff }}(\mathrm{mm})$ & 1.0294 & 1.0342 \\
\hline
\end{tabular}

Table 3: FBGs characteristics for $L_{g}=2 \mathrm{~mm}$

\begin{tabular}{|c|c|c|c|}
\cline { 3 - 4 } \multicolumn{2}{c|}{} & PMMA FBG & Silica FBG \\
\hline \multirow{3}{*}{$\lambda=850 \mathbf{n m}$} & $\mathrm{r}$ & 0.8116 & 0.8121 \\
\cline { 2 - 4 } & $\mathrm{t}$ & 0.1878 & 0.1879 \\
\cline { 2 - 4 } & $\mathrm{L}_{\text {eff }}(\mathrm{mm})$ & 0.6095 & 0.6096 \\
\hline \multirow{3}{*}{$\lambda=1550 \mathbf{n m}$} & $\mathrm{r}$ & 0.4322 & 0.4489 \\
\cline { 2 - 4 } & $\mathrm{t}$ & 0.5305 & 0.5511 \\
\cline { 2 - 4 } & $\mathrm{L}_{\text {eff }}(\mathrm{mm})$ & 0.8223 & 0.8264 \\
\hline
\end{tabular}

The FBGs characteristics from Table 2 and Table 3 are used to investigate the stress sensitivity of a Fabry-Perot device when is formed with two uniform FBGs. Figure 2 shows the stress sensitivity of PMMA and silica based Fabry-Perot interferometers operating at $\lambda=850 \mathrm{~nm}$ with a grating length of $L_{g}=3 \mathrm{~mm}$ each. Despite the similar FBG characteristics for both PMMA and silica fibres (Table 2), the stress sensitivity of the PMMA Fabry-Perot starts to reduce as the interferometric cavity length increases beyond a certain point due to the attenuation along the cavity length. The maximum sensitivity occurs when the cavity length is $5.18 \mathrm{~cm}$ and beyond that point, sensitivity starts to decrease. Silica based Fabry-Perot sensitivity increases with cavity length because silica attenuation is negligible compared to PMMA. However, PMMA based Fabry-Perot exhibit higher stress sensitivity compared to silica despite the high loss due to the different material properties. Silica Fabry-Perot interferometers can reach the same stress sensitivity level when the cavity length is $44.83 \mathrm{~cm}$, which is a large sensor.

Figure 3 shows the same device operating at $\lambda=1550 \mathrm{~nm}$ where the attenuation in PMMA is much higher. This results in lower sensitivity levels with a maximum obtained sensitivity at $0.83 \mathrm{~cm}$ cavity length. However, when Fabry-Perot sensors are preferred to be constructed close to this size, PMMA is the right material to be used as it can offer enhanced stress sensitivity compared to silica based devices which reach the same sensitivity level with a cavity length of $4.82 \mathrm{~cm}$. 


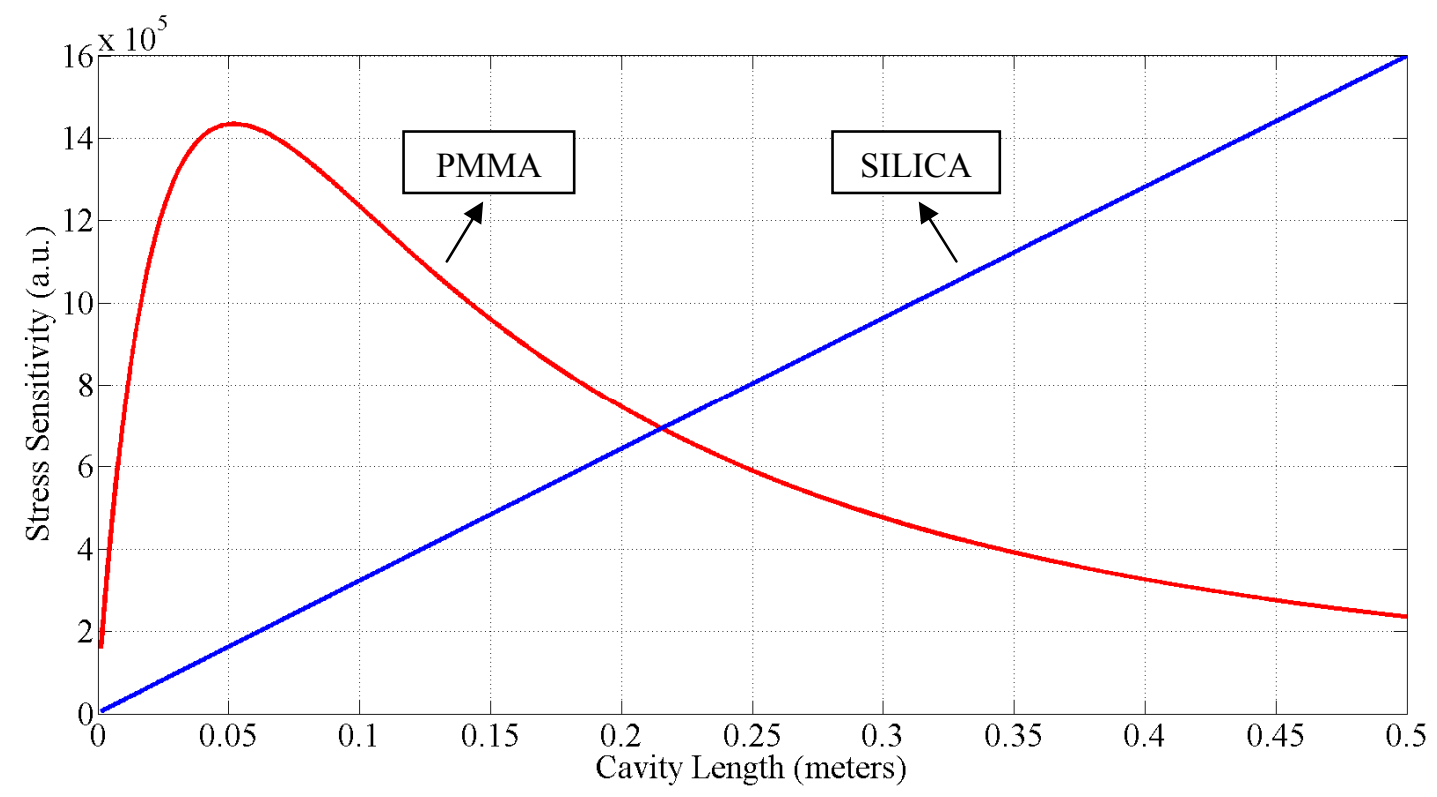

Figure 2: Stress sensitivity of Fabry-Perot with $L_{g}=3 \mathrm{~mm}$ operating at $\lambda=850 \mathrm{~nm}$

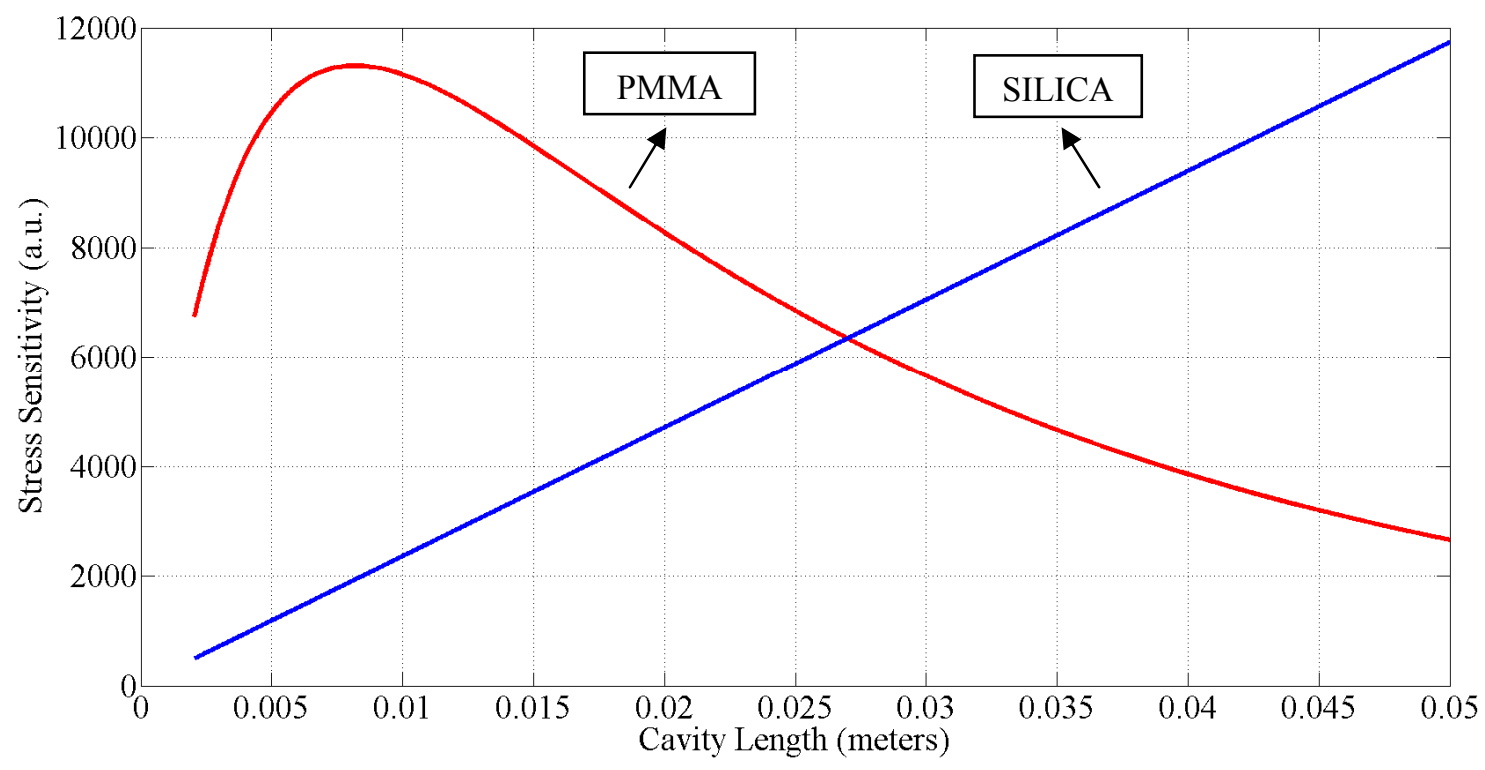

Figure 3: Stress sensitivity of Fabry-Perot with $L_{g}=3 \mathrm{~mm}$ operating at $\lambda=1550 \mathrm{~nm}$

Figure 4 and Figure 5 illustrate the same Fabry-Perot analysis, but with shorter grating lengths of $L_{g}=2 \mathrm{~mm}$ which means lower grating reflection coefficients. Both figures show that the reflection and transmission coefficients play an important role in Fabry-Perot performance. The interferometric sensors with grating lengths of $L_{g}=2 \mathrm{~mm}$ can obtain their maximum sensitivity levels with much longer cavity lengths. For example, the maximum sensitivity of the Fabry-Perot in Figure 2 occurs with a cavity length of $5.18 \mathrm{~cm}$ and similar sensitivity levels to the Fabry-Perot in Figure 4 which occurs with $22 \mathrm{~cm}$ cavity length due to the smaller reflection coefficients of the FBGs. Therefore, assessing grating reflection and transmission coefficients is essential during the fabrication of POF based Fabry-Perot sensors. 


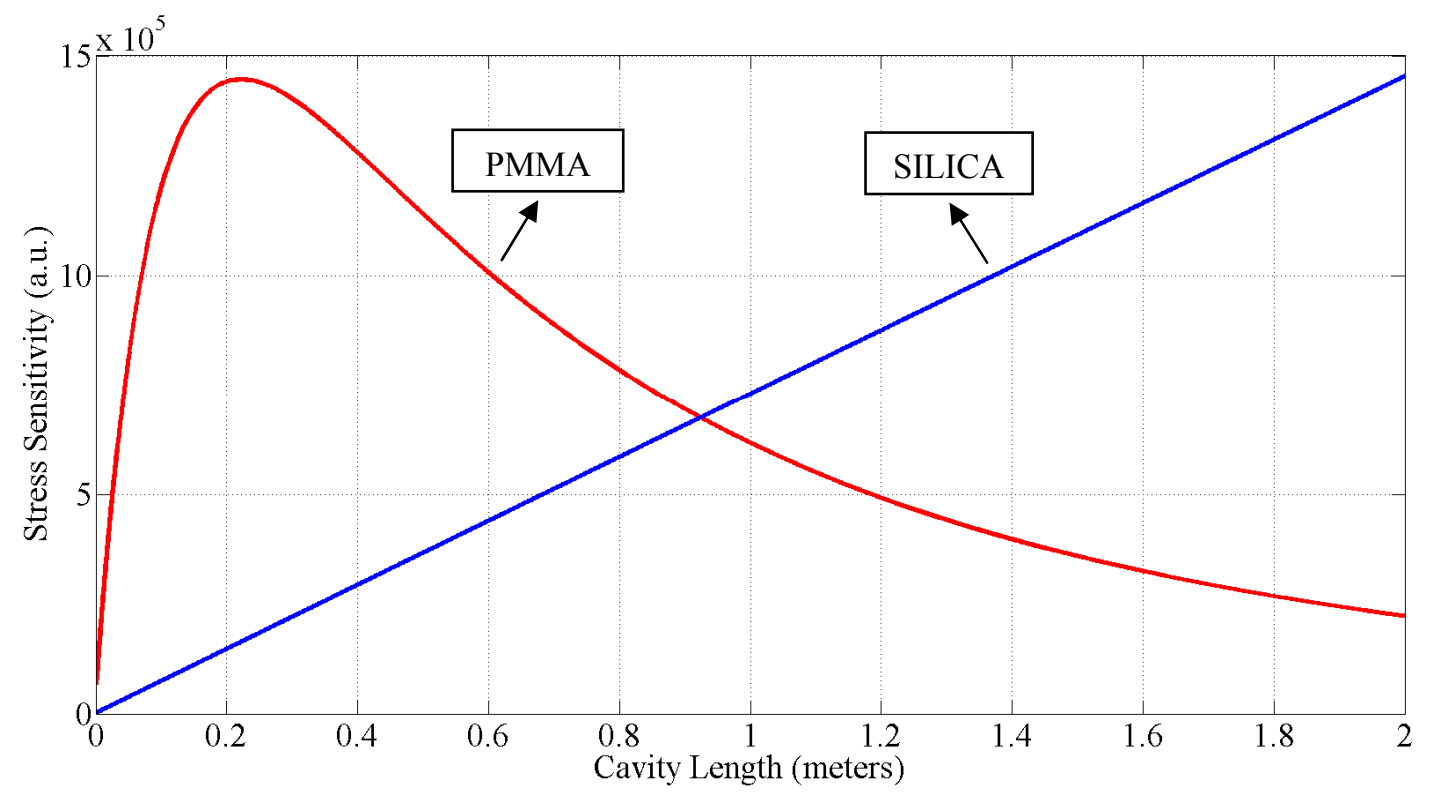

Figure 4: Stress sensitivity of Fabry-Perot with $L_{g}=2 \mathrm{~mm}$ operating at $\lambda=850 \mathrm{~nm}$

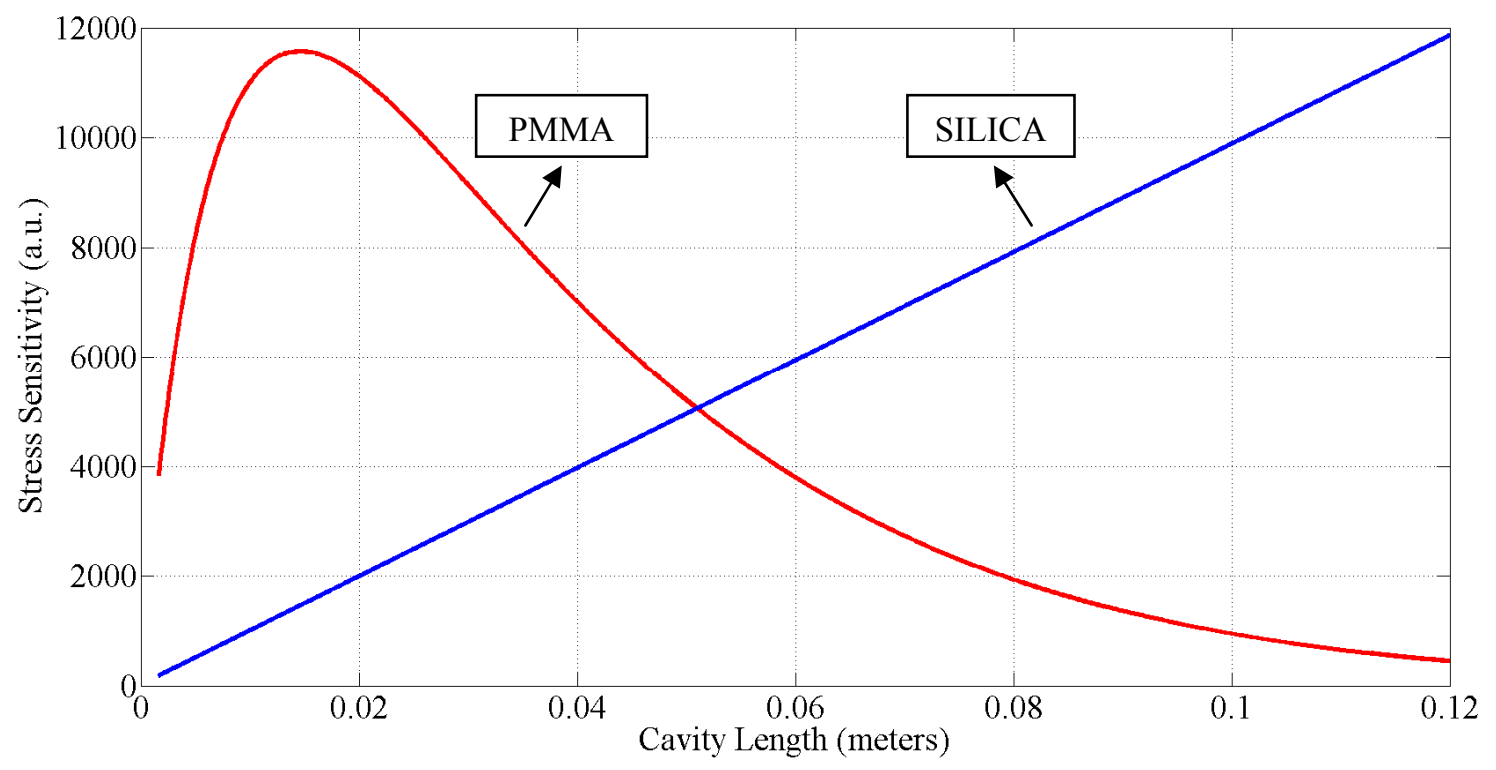

Figure 5: Stress sensitivity of Fabry-Perot with $L_{g}=2 \mathrm{~mm}$ operating at $\lambda=1550 \mathrm{~nm}$

\section{CONCLUSION}

The performance analysis of POF based Fabry-Perot devices for stress sensing is presented. Two uniform Bragg gratings are considered in this model which act as mirrors and form a Fabry-Perot interferometric cavity. The gratings have finite length and their characteristics define the actual cavity length of the interferometer and its performance. A higher grating reflection coefficient provides enhanced stress sensitivity and the maximum obtained sensitivity of the interferometer can be reached with lower cavity lengths. The fibre loss also negatively affects the performance of the Fabry-Perot device. 
However, despite the high attenuation in PMMA fibre, the analysis in this paper shows that the stress sensitivity of POF based Fabry-Perot sensors is higher in some regimes than the one in silica fibre, due to their different material properties. Therefore, analysing and finding the optimum conditions for maximum performance of Fabry-Perot sensors is essential when they are fabricated in POFs.

\section{ACKNOWLEDGMENT}

The research leading to these results has received funding from the People Programme (Marie Curie Actions) of the European Union's Seventh Framework Programme FP7/2007-2013/ under REA grant agreement No. 608382.

\section{REFERENCES}

[1] D. J. Webb, "Fibre Bragg grating sensors in polymer optical fibres", Measurement Science and Technology, 26(9), 092004 (2015).

[2] J. Brandrup, E. H. Immergut, E. A. Grulke et al., [Polymer handbook] Wiley New York, (1999).

[3] M. Silva-Lopez, A. Fender, W. N. Macpherson et al., "Strain and temperature sensitivity of a single-mode polymer optical fiber", Optics Letters, 30, 3129-3131 (2005).

[4] M. C. J. Large, J. Moran, and L. Ye, "The role of viscoelastic properties in strain testing using microstructured polymer optical fibres (mPOF)", Measurement Science \& Technology, 20, (2009).

[5] S. Kiesel, P. Van Vickle, K. Peters et al., "Intrinsic polymer optical fiber sensors for high-strain applications", Proc. SPIE 6167, 616713-616713-11 (2006).

[6] T. X. Wang, Y. H. Luo, G. D. Peng et al., "High-sensitivity stress sensor based on Bragg grating in BDK-doped photosensitive polymer optical fiber", 8351 (2012).

[7] C. Marques, A. Pospori, D. Sáez-Rodríguez et al., "Fiber optic liquid level monitoring system using microstructured polymer fiber Bragg grating array sensors: performance analysis", International Society for Optics and Photonics 9634, 96345V-4 (2015).

[8] A. Stefani, S. Andresen, W. Yuan et al., "Dynamic Characterization of Polymer Optical Fibers", Ieee Sensors Journal, 12, 3047-3053 (2012).

[9] F. Bischoff, "Organic polymer biocompatibility and toxicology", Clinical chemistry, 18(9), 869-894 (1972).

[10] T. Watanabe, N. Ooba, Y. Hida et al., "Influence of humidity on refractive index of polymers for optical waveguide and its temperature dependence", Applied Physics Letters, 72, 1533-1535 (1998).

[11] W. Zhang, D. J. Webb, and G. D. Peng, "Investigation Into Time Response of Polymer Fiber Bragg Grating Based Humidity Sensors", Journal of Lightwave Technology, 30, 1090-1096 (2012).

[12] R. M. Waxler, and G. Cleek, "Effect OF temperature and pressure on refractive-index of some oxide glasses", Journal of Research of the National Bureau of Standards, Section A: Physics and Chemistry(6), 755-763 (1973).

[13] L. Eldada, and L. W. Shacklette, "Advances in polymer integrated optics", Selected Topics in Quantum Electronics, IEEE Journal of, 6(1), 54-68 (2000).

[14] Z. Zhang, P. Zhao, P. Lin et al., "Thermo-optic coefficients of polymers for optical waveguide applications", Polymer, 47, 4893-4896 (2006).

[15] H. B. Liu, H. Y. Liu, G. D. Peng et al., "Strain and temperature sensor using a combination of polymer and silica fibre Bragg gratings", Optics Communications, 219, 139-142 (2003).

[16] X. S. Cheng, W. W. Qiu, W. X. Wu et al., "High-sensitivity temperature sensor based on Bragg grating in BDK-doped photosensitive polymer optical fiber", Chinese Optics Letters, 9, (2011).

[17] A. Yariv, "Coupled-mode theory for guided-wave optics", Quantum Electronics, IEEE Journal of, 9(9), 919-933 (1973).

[18] T. Erdogan, "Fiber grating spectra", Lightwave Technology, Journal of, 15(8), 1277-1294 (1997).

[19] Y. O. Barmenkov, D. Zalvidea, S. Torres-Peiró et al., "Effective length of short Fabry-Perot cavity formed by uniform fiber Bragg gratings", Optics Express, 14(14), 6394-6399 (2006).

[20] C. D. Butter, and G. B. Hocker, "Fiber optics strain gauge", Applied Optics, 17(18), 2867-2869 (1978).

[21] J. G. A. Griffiths, "Tables of physical and chemical constants. By G. W. C. Kaye and T. H. Laby", The Analyst, 73(873), 704 (1948). 
[22] R. M. Waxler, D. Horowitz, and A. Feldman, "Optical and physical parameters of Plexiglas 55 and Lexan", Applied Optics, 18(1), 101-104 (1979).

[23] A. Bertholds, and R. Dandliker, "Determination of the individual strain-optic coefficients in single-mode optical fibres", Lightwave Technology, Journal of, 6(1), 17-20 (1988).

[24] D. D. Raftopoulos, D. Karapanos, and P. S. Theocaris, "Static and dynamic mechanical and optical behaviour of high polymers", Journal of Physics D: Applied Physics, 9(5), 869 (1976).

[25] H. P. A. Van den Boom, W. Li, P. K. Van Bennekom et al., "High-capacity transmission over polymer optical fiber", IEEE Journal of Selected Topics in Quantum Electronics, 7(3), 461-470 (2001).

[26] I. H. Malitson, "Interspecimen Comparison of the Refractive Index of Fused Silica", Journal of the Optical Society of America, 55(10), 1205-1208 (1965).

[27] N. Sultanova, S. Kasarova, and I. Nikolov, "Dispersion properties of optical polymers", Acta Physica Polonica A, 116(4), 585-587 (2009). 